\title{
Do good self-managers have less physical and social resource deficits and more well-being in later life?
}

\author{
Nardi Steverink $\cdot$ Siegwart Lindenberg
}

Published online: 20 August 2008

(C) The Author(s) 2008. This article is published with open access at Springerlink.com

\begin{abstract}
Proactive self-management is likely to be part of resource maintenance and well-being in later life, but empirical evidence is scarce. Therefore, we investigated (a) whether self-management ability (SMA) is associated with lower resource deficits, and (b) whether it is related directly and indirectly to life satisfaction (LS), positive affect (PA) and negative affect (NA). Regression and mediational analyses $(N=439$, aged 65 years and older), showed that SMA related to resource deficits, and had direct associations with the three well-being indicators. Indirect associations-via lower resource deficits-were found for LS, whereas the association between SMA and PA was mostly a direct one, being mediated only to a small extent by physical resource deficits. The association between SMA and NA was mediated only by social resource deficits. It is concluded that better SMA seems to matter to resource maintenance and well-being, but future research needs to unravel the differential findings for physical and social resource deficits and for the separate well-being indicators.
\end{abstract}

Keywords Resources - Self-management ability · Life satisfaction - Positive affect $\cdot$ Negative affect

\footnotetext{
N. Steverink $(\bowtie)$

Department of Health Sciences, Section Health Psychology, University Medical Centre Groningen, University of Groningen, Antonius Deusinglaan 1, 9713 AV Groningen, The Netherlands e-mail: b.j.m.steverink@med.umcg.nl

N. Steverink $\cdot$ S. Lindenberg

Interuniversity Center for Social Science Theory and

Methodology (ICS), University of Groningen,

Grote Rozenstraat 31, 9712 TG Groningen, The Netherlands
}

\section{Introduction}

Since decades it is a generally shared assumption that individuals actively produce and regulate their own development and ageing (Lerner and Busch-Rossnagel 1981). Such active self-regulation is not only assumed in general models on self-regulation (e.g., Baumeister and Vohs 2004), but also in models on ageing-related selfregulation. For example, Baltes and Baltes (1990), in their SOC model, distinguish three components of adaptive behaviour in developmental change across the life span: selection, optimization, and compensation. Heckhausen and Schulz (1995), in their life-span theory of control, consider primary and secondary control strategies having selective and compensatory functions in developmental regulation. As a third example, Rothermund and Brandtstädter (2003), in their dual-process model of ageingrelated coping, consider assimilative modes of coping (i.e., attempts to avoid or diminish actual or anticipated losses by instrumental, self-corrective, and compensatory activities) and accommodative processes (devaluation of, or disengagement from, blocked goals and a lowering of personal performance standards and aspirations).

Roughly, the active and adaptive processes that are identified in these models can be regarded in terms of two broad areas of behaviour: as reactive (i.e., as a reaction to a change, obstacle or loss in resources) and as proactive (i.e., prior to a change, obstacle or loss). However, although most models do consider both kinds of behaviours theoretically, the empirical focus of most approaches has been on the reactive loss-buffering function of these strategies. For example, primary and secondary control strategies (i.e., persistence, positive reappraisals, and lowering aspirations) were investigated in relation to subjective well-being in individuals who experienced health or financial stress 
(Wrosch et al. 2000). Rothermund and Brandtstädter (2003) investigated coping with deficits and losses in later life and established the importance of accommodative processes (e.g., lowering of personal comparison standards and downgrading of the importance of goals). In a study based on the SOC-model (Baltes and Baltes 1990) it was investigated whether the life-management strategies of selection, optimization and compensation play a role in ageing satisfaction by buffering the effect of low resources (Jopp and Smith 2006).

Undoubtedly, loss-related self-management is important in the process of ageing. Still, (pro)active self-management may be equally important for ageing well, and the question that is largely left unanswered so far is what active selfmanagement means for resource building and well-being, not just for dealing with losses or deficits. It seems likely that an important part of the self-management process is that people do not just wait for losses to occur or goals to get blocked, but, instead, try to build and maintain important resources for well-being (cf. Aspinwall and Taylor 1997; Hobfoll 2002). Active self-management has been found in the area of health, showing proactive and preventive selfmanagement behaviours for physical resources in the oldold, such as exercising and dieting (Kahana et al. 2002). Also the study of Jopp and Smith (2006) — although their focus was on the protective role of SOC strategies in resource-poor old people-indicated that SOC had positive associations with resources, and an independent effect on well-being in the young-old. Bode et al. (2007) investigated aspects of proactive coping in middle and late adulthood, but this study did not specifically address the question whether proactive coping is actually associated with fewer resource losses and with well-being indicators.

In this paper we argue that self-management in ageing may also imply active strategies or abilities that are not only applied in reaction to loss, but also as active behaviours aimed at taking care of one's important resources for well-being. More insight in these active resource maintenance behaviours of ageing individuals may be an important clue for policy and practice aimed at the prevention or delay of resource losses in older people.

The aim of this study is to contribute to the knowledge about (pro)active self-management in ageing, by proposing to consider ageing-related self-management ability with a more explicit focus on the active management of resources. The rationale for our approach is the idea that, if selfmanagement ability indeed also is important in a proactive sense, then people having higher levels of self-management ability should have gained and maintained higher levels of resources and thus have lower levels of resource deficits. This, in turn, should have a positive impact on well-being, as proposed by most resource theories (e.g., Hobfoll 2002). In addition to this resource-mediated effect on well-being, higher levels of self-management ability may also be related directly to indicators of well-being. Self-management ability may be closely related to control, and control and subjective well-being are consistently found to be positively associated (Lachman and Prenda Firth 2004; Thompson 2002).

In order to address these questions empirically, we use a recently developed model of self-management in the process of ageing that considers self-management ability as the orchestrated use of various active self-management abilities. According to this model, which we have called the theory of Self-Management of Well-being (Steverink et al. 2005), the achievement and maintenance of wellbeing over the life-span depends on whether people have adequate resources for fulfilling their well-being needs and goals, and, more importantly, whether they have the skills or abilities to manage these resources such that these resources are indeed achieved and maintained, and eventually compensated or restored during the life-span. Thus, overall self-management ability is defined as a generative capacity (consisting of several sub-abilities) to take care of one's own important resources, i.e., resources that contribute to well-being. Note that this conceptualization of SMA explicitly deals with functional behaviours and cognitions (i.e., abilities) that are linked to basic needs, the fulfilment of which is fundamental for well-being (i.e., comfort, affection-see Steverink et al. 2005 for a detailed explanation of this idea).

Let us take the example of friendship, as a resource for affection, to illustrate this link and explicate the six subabilities that together make up overall SMA. Note that we could also have used the example of, for instance, physical fitness as a resource for comfort, the latter being a physical need which fulfilment is important for physical well-being.

Prerequisites in achieving and maintaining friends are the ability to take initiatives in making friends and the ability to be self-efficacious with regard to one's own behaviour in making friends and being a friend. The maintenance of a friendship furthermore requires the ability to invest in the friendship, which, in turn, is helped by the ability to have a positive frame of mind with regard to this friendship in the future. Moreover, there is a self-management ability that helps to create synergetic effects and thus optimize the outcome of friendship for well-being: The ability to achieve and maintain multifunctionality in a friendship. A multifunctional friend is a person who can satisfy one's need for affection, but at the same time supports the fulfilment of other important needs such as the need for stimulation, e.g., by jointly participating in interesting activities. The underlying assumption is that the synergetic effects created by mutually reinforcing activities yield more overall well-being than unifunctional activities (Nieboer and Lindenberg 2002). Finally, there is a self-management ability that 
reduces the negative effects of loss on well-being: The ability to take care of variety with regard to friendship. Ensuring variety simply means not putting all of one's egg in one basket, i.e., to have a variety of friends. If something happens to one friendship, there are others to buffer the negative effect.

Although each of the six sub-abilities is considered important in itself, it is the combined and orchestrated use of all six what makes people better self-managers in the process of achieving and maintaining higher levels of resources for well-being in the process of ageing. This is in line with the approach of the life-management strategies based on the SOC model (Freund and Baltes 1998, 1999) in which the combined use of S- O- and C-strategies together makes up adaptive life management. Yet, the linking to concrete basic needs distinguishes our approach from most other models, since the latter mostly incorporate only abstract goals and plans [see, e.g., the operationalisation of the SOC-model, in Freund and Baltes (1998)].

The use of the six abilities will cumulate to produce higher levels of overall self-management ability, because the combined use of the six is likely to lead to mutual reinforcement. For example, people who are more selfefficacious often will also take more initiatives, and may be better able to invest, which again may lead to reinforcement spirals. The confirmatory factor-analyses that were executed in the development studies of a scale measuring this orchestrated concept of self-management ability showed that, indeed, overall self-management ability could be measured reliably as a composite concept of interrelated abilities (see Schuurmans et al. 2005).

The aim of the present study is to test three hypotheses based on the arguments above. First, because SMA, as a generative capacity, may help to achieve and maintain important resources, higher levels of self-management ability are expected to relate positively to lower levels of physical and social resource deficits (H1). Secondly, because SMA ultimately is directed at the management of resources for well-being, higher levels of self-management ability are expected to relate positively to indicators of subjective well-being (H2). Thirdly, because SMA helps to prevent resource deficits, and because lower levels of resource deficits contribute to higher levels of well-being, we expect that the association between SMA and wellbeing indicators is mediated, at least in part, by lower levels of physical and social resource deficits (H3).

Note that it is likely that the process proposed here is a self-reinforcing mechanism, indicating that higher levels of well-being may again feed back to higher levels of SMA, and so again feed back to higher levels of resources, etc. It may also be the case that SMA helps to buffer the effects of resource loss on well-being. This is the commonly investigated loss-buffering or moderating role of self-management or self-regulation. However, we explicitly propose here to test a possible meditational mechanism, trying to answer the question whether higher levels of SMA relate to lower levels of resource deficits, and therefore to higher levels of wellbeing.

\section{Methods}

Sample and procedure

In August 2001 a questionnaire was sent to a random sample of 1,000 community-dwelling persons 65 years of age and older, living in the North of the Netherlands. The addresses were randomly drawn from the registers of six municipalities, consisting of smaller and larger villages and cities, and having an average income comparable to the national mean.

Of these 1,000 addressees, $44.0 \%$ returned the completed questionnaire, resulting in a sample of $N=439$. Although the response rate might seem low, and there is the potential danger of non-response bias, it is similar (Picavet 2001), or even quite high (Buttle and Thomas 1997), compared to that in similar studies in which the respondents received a questionnaire by mail. Moreover, a comparison of the sample with the general population of people aged 65 years and older in the Netherlands (CBS 2003) showed that the sample contained comparable proportions of males $(42 \%)$ to females $(58 \%)$ and people living alone (34\%). With regard to age, however, it was found that the sample contained a smaller proportion of people aged 85 and older (5\%) as compared to the proportion of people aged 85 and older in the general population of people aged 65 and older (10\%). Thus, the oldest-old seem to be somewhat underrepresented in our sample.

The average age of the study participants was 74.25 years $(\mathrm{SD}=6.56)$, with a range from 65 to 97 years. Marital status: $68.6 \%$ were married or having a partner, $31.4 \%$ were single. Ninety-four percent lived independently in the community, whereas $6 \%$ lived in a sheltered living arrangement (not nursing homes). Level of education: $25,8 \%$ had only 7 years of education or less (i.e., only primary school or less), $52,2 \%$ had about 10 years of education, $18.7 \%$ had about $12-16$ years of education, and $3.3 \%$ had 18 years of education or more (i.e., university level).

Measures/instruments

\section{Self-management ability}

Self-management ability was measured by the self-management ability scale (SMAS-30; Schuurmans et al. 2005). 
This scale is based on the conceptualization of self-management ability as proposed by the self-management of well-being theory (see "Introduction"). It contains 30 items, covering all aspects of the theoretical idea of subabilities linked to well-being needs (for details see Steverink et al. 2005). The phrasing of all items and details on the sub-abilities can be found in Schuurmans et al. (2005). In principle, the sub-abilities can be measured separately, but this is meant primarily for measuring the effectiveness of manipulations of each of the six sub-abilities in SMA intervention studies (see Frieswijk et al. 2006; Kremers et al. 2006). Essentially, self-management ability is represented by the orchestrated use of all abilities, which together make up overall SMA. Therefore, in the present study the composite score of overall SMA was used. Sample items are: "How often do you take the initiative to keep yourself busy?"; "Do you keep busy with the things you are good at so that you stay good at them?". Answer categories are on a 5 or 6 point Likert scale, which are transformed such that the overall scale score ranges from 0 to 100 . Scores are summed such that higher scores represent higher SMA. It is important to note that the items of the SMA scale are phrased in terms of active behaviours and/or active cognitions (i.e., SMA is defined as being actively engaged in taking care of one's own overall wellbeing). As such the conceptualization of SMA explicitly differs from the other constructs used here (e.g., social deficits, well-being indicators), because the latter measure cognitive or affective evaluations of experiences or situations instead of active engagement. The scale has been tested in several studies and has shown good psychometric properties in terms of reliability and validity as well as in terms of good model fit in confirmatory factor analyses (for details see Schuurmans et al. 2005). Especially the latter underscored the theoretical basis of the six abilities making up one overall construct of SMA. Internal consistency (Cronbach's alpha) of the scale in this study was 0.92 .

\section{Physical deficits}

The deficit of physical resources was measured by asking the respondents to indicate whether they had problems (yes or no) in each of nine domains of physical functioning. These domains included various aspects of both ADL and IADL [e.g., shopping, walking outside the home, (un)dressing, and toileting], and aspects of energy, and of morbidity (suffering from more than one chronic disease). Samples items are "Can you do the shopping yourself?", "Can you fully independently dress and undress yourself?", The responses were summed and coded such that a higher score indicates more physical deficits. Internal consistency (Cronbach's alpha) of the scale in this study was 0.67 .

\section{Social deficits}

The deficit of social resources was measured by three items asking the respondents whether they felt deficits regarding social relationships with others. These three items are derived from a widely used scale measuring socio-emotional isolation (De Jong Gierveld and Kamphuis 1985). Sample items are "Do you miss having people around?", "Do you often feel rejected?". Answer categories were "no", "sometimes", and "yes". Scores were summed such that higher scores indicated a higher level of social deficits. Internal consistency of the scale in this study was 0.76 .

\section{Subjective well-being}

As is often done (Lucas et al. 1996) we measured one cognitive and two affective components of well-being. Life satisfaction (LS) is seen as the result of a cognitive evaluation of one's life as a whole and was measured with the five-items Satisfaction With Life Scale (Diener et al. 1985). A sample item is "In most ways, my life is close to my ideal". Answers on a 5-point scale ranged from "strongly disagree' to 'strongly agree'. Scores were summed such that higher scores represented higher LS. Cronbach's $\alpha$ in this study was 0.85 . Affect is considered as the presence of positive mood, and the absence of negative mood. For this we used the Positive and Negative Affect Scale (PANAS), which consists of two 10-item scales (Watson et al. 1988). Sample items are: "During the past few months, how often did you feel... excited, enthusiastic, alert, inspired?" (Positive Affect-PA) and "... sad, upset, afraid, nervous, scared?" (Negative Affect-NA). Answer categories ranged from 'never' to 'very often' on a 5-point scale. Scores were summed such that higher scores indicate higher PA and higher NA. Internal consistency coefficients in the present study were 0.83 and 0.85 for PA and NA, respectively.

\section{Level of education}

Level of education was addressed in this study in order to control for possible confounding effects of level of education on level of self-management ability. It was measured by one question asking what the highest level of education is that the respondent has ever finished. Answer categories ranged from no or only basic education (less than 7 years of education) to university level (more than 18 years of education) on a 6-point scale.

\section{Analysis}

All three hypotheses were tested by applying multiple regression analyses. In all regression models, age, gender 
and level of education were controlled for, because-as can be seen in Table 1 -there are moderate associations between these variables and most of the main study variables. The mediation effects in the third hypothesis were tested by using the four-step mediational method as proposed by Baron and Kenny (1986). Step 1: show that the predictor variable is correlated with the criterion variable. This step establishes that there is an effect that may be mediated. Step 2: show that the predictor variable is correlated with the mediator. This step essentially involves treating the mediator as if it were a dependent variable. Step 3: show that the mediator affects the criterion variable. Here the predictor variable must be controlled in establishing the effect of the mediator on the criterion variable. Step 4: To establish that the mediator completely mediates the relationship between the predictor and the criterion variable, the effect of the predictor on the criterion variable controlling for the mediator should be zero. If all four of these steps are met, then the data are consistent with the hypothesis that the mediator completely mediates the relationship between the predictor and the criterion variable. If the first three steps are met but step 4 is not, then partial mediation is indicated. The amount of mediation is defined as the reduction of the effect of the predictor on the criterion variable.

Finally, we tested the degree of mediation by using a version of a test originally proposed by Sobel (1982) and modified by Baron and Kenny (1986; see also Kenny et al. 1998).

\section{Results}

Table 1 presents the descriptives and the Pearson correlations of all variables.

In Table 2 the results of two separate regression analyses are shown, covering the test of the first hypothesis (H1).
This hypothesis stated that higher levels of self-management ability relate to lower levels of physical and social deficits. In each model, self-management ability is entered, together with the control variables age, gender and education. Moreover, in the model for physical deficits the amount of social deficits is controlled for, whereas in the model for social deficits the amount of physical deficits is controlled for.

As can be seen in Table 2, level of overall self-management ability is negatively related to both physical and social deficits, as we expected. Yet, the coefficient of selfmanagement ability is substantially larger for physical deficits than for social deficits, indicating that self-management ability plays a stronger role in the former. This is also visible in the larger amount of variance accounted for: $27 \%$ for physical deficits versus $14 \%$ for social deficits. With regard to the control variables, we also see that age has a stronger association with physical deficits than with social deficits. On the other hand, gender is only significantly related to social deficits, not to physical deficits, and level of education is only marginally significant (negatively) for physical deficits. It can be concluded that the first hypothesis is supported: higher levels of self-management ability relate to lower levels of both physical and social deficits.

The results of the second and third hypotheses are shown in Table 3, in three separate regression models, with life satisfaction, positive affect, and negative affect as three dependent variables, respectively.

The second hypothesis stated that higher levels of selfmanagement ability relate to higher levels of subjective well-being, or more specifically: to higher levels of life satisfaction and positive affect, and lower levels of negative affect. Results of the test of $\mathrm{H} 2$ are shown in the first step of each of the three models, showing that, as expected, self-management ability is positively related to life satisfaction and to positive affect, and negatively to negative

Table 1 Descriptives and Pearson Correlations of All Variables

\begin{tabular}{|c|c|c|c|c|c|c|c|c|c|c|c|}
\hline & \multicolumn{3}{|c|}{ Descriptives } & \multicolumn{8}{|c|}{ Correlations } \\
\hline & Range & $M$ & SD & 1 & 2 & 3 & 4 & 5 & 6 & 7 & 8 \\
\hline 1 Age & $65-97$ & 74.25 & 6.56 & & & & & & & & \\
\hline 2 Gender $(0=$ male $)$ & $0-1$ & 0.58 & 0.49 & 0.01 & & & & & & & \\
\hline 3 Education & $1-6$ & 2.66 & 1.44 & $-0.11^{*}$ & $-0.26^{* *}$ & & & & & & \\
\hline 4 Self-management ability & $2-96$ & 62.58 & 12.83 & $-0.30 * *$ & 0.08 & $0.18 * *$ & & & & & \\
\hline 5 Physical resource deficits & $0-7$ & 0.92 & 1.25 & $0.39 * *$ & 0.08 & $-0.20 * *$ & $-0.43 * *$ & & & & \\
\hline 6 Social resource deficits & $0-6$ & 1.36 & 1.65 & $0.21 * *$ & $0.14 * *$ & $-0.16^{* *}$ & $-0.29 * *$ & $0.25 * *$ & & & \\
\hline 7 Life satisfaction & $0-20$ & 14.25 & 3.65 & $-0.11^{*}$ & $-0.11^{*}$ & $0.14 * *$ & $0.47 * *$ & $-0.37 * *$ & $-0.47 * *$ & & \\
\hline 8 Positive affect & $3-38$ & 23.24 & 4.86 & $-0.23 * *$ & 0.04 & $0.21 * *$ & $0.66 * *$ & $-0.37 * *$ & $-0.15 * *$ & $0.32 * *$ & \\
\hline 9 Negative affect & $0-32$ & 12.60 & 5.09 & 0.02 & $0.17 * *$ & -0.08 & $-0.21 * *$ & $0.15^{* *}$ & $0.38 * *$ & $-0.40 * *$ & -0.06 \\
\hline
\end{tabular}

** $P<0.01 ; * P<0.05$ 
Table 2 Regression analyses for physical and social resource deficits $(N=439)$

\begin{tabular}{lcc}
\hline & \multicolumn{2}{l}{ Resource deficits } \\
\cline { 2 - 3 } & Physical & Social \\
\hline Age & $0.27^{* * *}$ & $0.11^{*}$ \\
Gender & 0.06 & $0.14^{* *}$ \\
Education & $-0.08 \#$ & -0.05 \\
Self-management ability & $-0.30^{* * *}$ & $-0.19^{* * *}$ \\
Physical deficits & & $0.11^{*}$ \\
Social deficits & $0.10^{*}$ & \\
$R^{2}$ & 0.269 & 0.135 \\
$F$ & $31.9^{* * *}$ & $13.5^{* * *}$ \\
\hline
\end{tabular}

Note. Only standardized coefficients (Beta) are shown

*** $P<0.001 ; * * P<0.01 ; * P<0.05 ;{ }^{*} P<0.10$

affect. With respect to the control variables the data show that for life satisfaction and negative affect gender makes a difference (women reporting lower levels of life satisfaction and higher levels of negative affect than men); for positive affect educational level shows a positive significant association. As is indicated by the amounts of variance accounted for, self-management ability plays a substantial role in both life satisfaction and positive affect (with 22 and $38 \%$, respectively). The role of self-management ability in negative affect, however, is much smaller (7\%).

It can be concluded that the second hypothesis is largely supported by the data. Self-management ability is associated in the expected directions with indicators of subjective well-being, especially for life satisfaction and positive affect, but also, although less strongly, with negative affect.

The results of the test of the third hypothesis are also shown in Table 3, in the second step of each of the three models. The third hypothesis stated that the association between self-management ability and well-being indicators is both a direct and an indirect one, the latter being a mediation via lower levels of physical and social deficits. The mediation effects of (lower) levels of physical and social deficits by (higher) levels of self-management ability when predicting subjective well-being (i.e., life satisfaction, positive and negative affect) are analysed by using the Baron and Kenny (1986) four-step mediational method.

As can be seen in the second model of all three regression analyses in Table 3, the coefficients of selfmanagement ability in each second model become smaller as compared to each first model step, after entering physical and social deficits as mediators. For life satisfaction both mediators (physical and social deficits) have a significant coefficient, while the coefficient of self-management ability decreases from 0.45 to 0.31 . For positive affect only physical deficits are significant and the coefficient of self-management ability decreases from 0.58 to 0.55 . For negative affect, finally, only social deficits are significantly associated, and the coefficient of self-management ability decreases here from -0.23 to -0.13 .

In order to determine whether the observed reductions in the beta coefficients of self-management ability for each of the three indicators of subjective well-being are significant reductions (when physical and social deficits are entered in the regression) we assessed the degree of mediation by using a version of a test originally proposed by Sobel (1982) and modified by Baron and Kenny (see also Kenny et al. 1998). Results indicated a significant reduction of the coefficient of self-management ability on life satisfaction, for both mediators: $z_{\text {physical deficits }}=3.42, P<0.001$, and $z_{\text {social deficits }}=3.63, P<0.001$. For positive affect the test showed a significant mediating association for physical deficits, $z=2.44, P<0.05$. For negative affect a significant mediating relationship was found for social deficits,

Table 3 Regression analyses for life satisfaction, positive affect and negative affect, with mediation $(N=439)$

\begin{tabular}{|c|c|c|c|c|c|c|}
\hline & \multicolumn{2}{|c|}{ Life satisfaction } & \multicolumn{2}{|c|}{ Positive affect } & \multicolumn{2}{|c|}{ Negative affect } \\
\hline & 1 & 2 & 1 & 2 & 1 & 2 \\
\hline Age & 0.02 & $0.13 * *$ & -0.05 & -0.02 & -0.05 & $-0.11 *$ \\
\hline Gender & $-0.14 * *$ & $-0.07 \#$ & 0.02 & 0.02 & $0.18 * * *$ & $0.13 * *$ \\
\hline Education & 0.02 & -0.02 & $0.10 *$ & $0.09 *$ & 0.00 & 0.03 \\
\hline Self-management ability & $0.45^{* * *}$ & $0.31 * * *$ & $0.58 * * *$ & $0.55^{* * *}$ & $-0.23 * * *$ & $-0.13 * *$ \\
\hline Physical deficits & & $-0.19 * * *$ & & $-0.12 * *$ & & 0.04 \\
\hline Social deficits & & $-0.35 * * *$ & & 0.03 & & $0.34 * * *$ \\
\hline$R^{2}$ & 0.217 & 0.364 & 0.381 & 0.391 & 0.074 & 0.179 \\
\hline$R^{2}$ change & & 0.147 & & 0.01 & & 0.106 \\
\hline$F$ & $30.0 * * *$ & $41.2 * * *$ & $66.7 * * *$ & $46.3 * * *$ & $8.7 * * *$ & $15.7 * * *$ \\
\hline$F$ change & & $50.0 * * *$ & & $3.8^{*}$ & & $27.8 * * *$ \\
\hline
\end{tabular}

Note: Only standardized coefficients (Beta) are shown

*** $P<0.001 ; * * P<0.01 ; * P<0.05 ;{ }^{\#} P<0.10$ 
$z=3.51, P<0.001$. It can be concluded that the third hypothesis is largely supported: indications are found for both direct and indirect (i.e., mediating) associations of physical and social deficits on the relationship between self-management ability and indicators of subjective wellbeing. However, these mediating associations were different for the three different indicators of well-being: both physical and social deficits mediated the association between self-management ability and life satisfaction, but for positive affect there was only a mediation by physical deficits and for negative affect there was only a mediation by social deficits. Moreover, although all these mediations were significant, the mediation on positive affect was rather small: the beta coefficient of self-management ability only reduced from 0.58 to 0.55 when entering physical deficits, and the amount of variance accounted for only increased with $1 \%$ (from 38 to $39 \%$ ). The reductions for life satisfaction and negative affect were larger, and the changes in $R^{2}$ were also substantially bigger: from 22 to $36 \%$ for life satisfaction, and from 7 to $18 \%$ for negative affect.

It can be concluded that, first, self-management ability affects all indicators of subjective well-being directly. Secondly, it affects all indicators of subjective well-being indirectly, but differentially for the separate well-being indicators and differentially for either physical resources or social resources or both. Self-management ability is indirectly related to life satisfaction via its negative association with both physical and social resource deficits; secondly, it is indirectly related to positive affect via its-relatively small—negative association with physical (not social) resource deficits; and finally, it is indirectly related to negative affect via its positive association with social (not physical) resource deficits.

\section{Discussion}

Is better self-management ability (SMA) associated with lower levels of resource deficits and with higher levels of well-being? To the best of our knowledge, this question has not been addressed empirically before. The answer we find is yes on both counts. Results showed, first, that, as we expected, SMA relates positively to life satisfaction and to positive affect, and negatively to negative affect. Secondly, also as expected, SMA is negatively related to physical and social resource deficits. Finally, the relationship between SMA and well-being indicators is partly mediated by lower levels of resource deficits.

Overall, these findings add to the existing knowledge on aging-related self-regulation and self-management in the sense that they underscore the proactive function of selfmanagement in aging, not only the reactive or loss-buffering function as is mostly investigated (e.g., Rothermund and Brandtstädter 2003; Wrosch et al. 2000). Nevertheless, some findings are not entirely as expected and need some further thought. First of all, the mediation is different for physical or social resource deficits and for the three wellbeing indicators. Only the association between SMA and life satisfaction was found to be mediated by both physical and social deficits, as expected, and the size of this partial mediation was substantial (added variance accounted for was $15 \%)$. However, the associations between SMA and the two affective aspects of well-being showed differential patterns regarding the expected mediations. The association between SMA and positive affect was mediated by physical, not social deficits and the association between SMA and negative affect was mediated by social, not physical deficits. Also the sizes of these indirect associations showed differential patterns: for positive affect the added variance accounted for was only $1 \%$, whereas for negative affect it was $11 \%$, indicating that the association between self-management ability and positive affect is mostly a direct one.

What do these findings mean? On the one hand, our findings are in line with existing insights that show that positive and negative affect do not depend on the same factors and that an asymmetry exists between the two (e.g., Charles et al. 2001; Steverink and Lindenberg 2006; Watson et al. 1988). On the other hand, our results do not seem to converge with other insights. We found that positive affect especially relates to physical deficits, and negative affect to social deficits, whereas, e.g., Lawton (1984) found that positive affect was better predicted by exterior environmental transactions among which are interactions with friends, and negative affect by intrapersonal factors among which is functional health. The latter findings thus seem to oppose ours and it remains uncertain why this is so. Future research should shed more light on these intriguing findings.

A second remarkable finding is the strong direct-and almost absence of indirect-association between SMA and positive affect, as compared to the less strong direct—but stronger indirect-associations between SMA and both life satisfaction and negative affect. How can this difference be interpreted? A rather simple explanation could be that SMA, as we assume in the theory section, engenders a feeling of control, which, in turn, may contribute to being alert and elated, which are components of positive affect. A more elaborate but related explanation may be found in models that explicitly relate self-regulation processes to different affect dimensions. For example, Carver and Scheier (1998) point to processes of approach and avoidance and how these relate differentially to emotional responses. A related model (Higgins 1997) points to promotion and prevention focused processes with comparable emotional responses. Approach and promotion-focused 
processes represent a concern with gain or growth, whereas avoidance and prevention-focused processes reflect a concern with protection and safety. Doing well in approach or promotion is assumed to lead to feelings of elation and eagerness (cf. positive affect) and doing poorly to sadness and depression (cf. negative affect). On the other hand, doing well in avoidance or prevention is assumed to lead to relief and calmness, doing poorly to fear and anxiety (cf. negative affect). Relief and calmness may relate especially to the cognitive dimension of well-being, i.e., life satisfaction. In light of this theoretical perspective, it seems that our data, by relating self-management ability only to resource deficits, in fact only addressed aspects of avoidance or prevention, not aspects of approach or promotion. As a result, only the emotional correlates of avoidance and prevention could be found: Doing well (by self-management ability) at avoidance of resource deficits enhances feelings of relief and calmness, and thus life satisfaction, and doing poorly enhances feelings of fear and anxiety and thus negative affect. Following this line of reasoning, one can hypothesize that indicators of doing well (by selfmanagement ability) at resource gain or growth should be associated with elation and eagerness (positive affect). Because the data showed a strong direct association between self-management ability and positive affect, it seems that self-management ability-as conceptualized here-indeed incorporates approach and promotion focused aspects (in addition to avoidance and prevention focused aspects). However, because resource growth was not measured and therefore could not be included in the analyses as a mediator, the indirect association between self-management ability and positive affect via resource growth could not be tested. Future research should explicitly measure not only whether self-management ability is associated with fewer resource deficits, but also whether it is associated with more resource growth or gain, in order to get more insight in the possible approach or promotion-focused aspects of self-management ability.

Our study also has some limitations. The first and most important limitation is that we only had cross-sectional data available. Although we based the analysis on clear theoretical reasoning (predicting the direction of causality and mediation as we did), the existence of other possible causal directions can not be ruled out by cross-sectional data. It may, e.g., also be possible that physical and social deficits (and also lower levels of well-being) have a negative influence on self-management ability. It even seems plausible that reinforcing spirals and feedback loops exist between self-management ability, resources and well-being indicators. This can only be tested reliably on the basis of longitudinal data, that, moreover, cover a time-span long enough to make such feedback processes visible. Still, to check for the possibility that alternative relationships exist between the variables considered here, we conducted some additional analyses to increase our confidence that, as hypothesized, resource deficits partially mediated the relationship between SMA and well-being indicators. We performed two sets of additional analyses, again with the four-step meditational analyses as proposed by Baron and Kenny (1986). First, we examined SMA as mediating variable (instead of resource deficits). Second, we examined a completely opposite model, in which SMA was the outcome, the well-being indicators the predictors, and resource deficits the mediators. Results of these alternative analyses indeed showed some alternative relationships between the variables and also a few indications for partial mediation. However, all alternative models, except the model in which PA was the outcome and SMA the mediator, seemed less strong than the original models in terms of variance accounted for. In all alternative models the added variance accounted for (after including the mediators) ranged only from 1 to $7 \%$, whereas in the original models this was 15 and $11 \%$, respectively, for LS and NA (after including the mediators). Only the alternative model in which PA was the outcome, and SMA the mediator (instead of PD), seemed stronger than the original model (in which physical deficits was the mediator): now the added variance accounted for was $23 \%$ (vs. only $1 \%$ in the original model). As already indicated, and now confirmed by these additional analyses, the relationships between SMA and PA seems to be primarily a direct one, and thus different than initially expected. On the other hand, the additional analyses also underscore our initial hypotheses regarding LS and NA, showing that SMA may play a role in the maintenance of resources and in well-being. Thus, on the basis of our data so far, we do not need to reject our hypotheses that good self-managers are likely to have an advantage in resource maintenance, and subsequently in higher levels of LS and lower levels of NA. This is a first finding that, however, needs to be supported or rejected by future longitudinal research. In addition, as said, more research is needed to unravel the intriguing findings regarding the strong direct associations between SMA and PA.

A second limitation, as already mentioned, is that the study did not contain resource gains as mediators and therefore may falsely suggest that there is no link of SMA to indicators of well-being via resource gains. This is an important issue that should be addressed in future research. Also, future research may incorporate objective measures of physical and social resource deficits, in addition to the subjective measures we applied. This could yield more insight into the possible role of self-management ability in the gain or loss of objective resources.

A final limitation may be the relative high non-response. Such a rate of non-response is common in large-scale 
population surveys of older people. However, it urges researchers to be cautious when generalizing the results. Still, this problem is probably limited as well, because our sample was largely comparable with the general population of people aged 65 and older on demographic indicators such as gender distribution and proportions of people living alone. Only regarding age the sample seemed to contain a smaller proportion of people aged 85 years and older than is found in the general population (see "Methods"). This may limit the interpretation of the results to a certain extent, because this age group probably has higher mean levels of resource deficits than younger age groups. On the other hand, probably this age group also has lower mean levels of SMA, as well as lower mean levels of subjective well-being. Together this may neutralise the possible selection biases again. Nevertheless, this caveat should be taken into account when interpreting the results.

In conclusion, this study shows that SMA matters. Its major contribution is the empirical demonstration that, for older people, SMA is positively associated with both fewer physical and social resource deficits and with well-being. It indicates that people are actively engaged in maintaining their important resources, not just in dealing with losses after the fact. Ultimately this knowledge may help to design SMA interventions (Frieswijk et al. 2006; Kremers et al. 2006) and as such be useful for policy and practice in supporting older people's active pursuit of their own wellbeing.

Acknowledgments This research was supported by Grant 041-91046 from the Netherlands Organization for Health Research and Development (ZonMw) and received support from the University of Groningen. We are very grateful to Roy Stewart and Anne Boomsma for their help and advice on the statistical analyses.

Open Access This article is distributed under the terms of the Creative Commons Attribution Noncommercial License which permits any noncommercial use, distribution, and reproduction in any medium, provided the original author(s) and source are credited.

\section{References}

Aspinwall LG, Taylor SE (1997) A stitch in time: self-regulation and proactive coping. Psychol Bull 121:417-436

Baltes PB, Baltes MM (1990) Psychological perspectives on successful aging: the model of selective optimization with compensation. In: Baltes PB, Baltes MM (eds) Successful aging: perspectives from the behavioural sciences. Cambridge University Press, New York, pp 1-34

Baron RM, Kenny DA (1986) The moderator-mediator variable distinction in social psychological research: conceptual, strategic, and statistical considerations. J Pers Soc Psychol 51:11731182

Baumeister RF, Vohs KD (eds) (2004) Handbook of self-regulation: research, theory and applications. The Guilford Press, New York/London
Bode C, De Ridder DTD, Kuijer RG, Bensing JM (2007) Effects of an intervention promoting proactive coping competencies in middle and late adulthood. Gerontol 47:42-51

Buttle F, Thomas G (1997) Questionnaire colour and mail survey response rate. J Mark Res Soc 39:625-626

Carver CS, Scheier MF (1998) On the self-regulation of behavior. Cambridge University Press, New York

Centraal Bureau voor de Statistiek (CBS) (2003) StatLine [Data file]. Centraal Bureau voor de Statistiek, Voorburg/Heerlen

Charles ST, Reynolds CA, Gatz M (2001) Age-related differences and change in positive and negative affect over 23 years. J Pers Soc Psychol 80:136-151

De Jong Gierveld J, Kamphuis FH (1985) The development of a Rasch-type loneliness scale. Appl Psychol Meas 9:289-299

Diener E, Emmons RA, Larsen RJ, Griffin S (1985) The satisfaction with life scale. J Personal Assessment 49:71-75

Freund AM, Baltes PB (1998) Selection, optimization, and compensation as strategies of life management: correlations with subjective indicators of successful aging. Psychol Aging 13:531-543

Freund AM, Baltes PB (1999) Selection, optimization, and compensation as strategies of life management: correction to Freund and Baltes (1998). Psychol Aging 14:700-702

Frieswijk N, Steverink N, Buunk BP, Slaets JPJ (2006) The effectiveness of a bibliotherapy in increasing the self-management ability of slightly to moderately frail older people. Pat Educ Couns 61:219-227

Heckhausen J, Schulz R (1995) A life-span theory of control. Psychol Rev 102:284-304

Higgins ET (1997) Beyond pleasure and pain. Am Psychol 52:12801300

Hobfoll SE (2002) Social and psychological resources and adaptation. Rev Gen Psychol 6:307-324

Jopp D, Smith J (2006) Resources and life-management strategies as determinants of successful aging: on the protective effect of selection, optimization, and compensation (SOC). Psychol Aging 21:253-265

Kahana E, Lawrence RH, Kahana B, Kercher K, Wisniewski A, Stoller E, Tobin J, Stange K (2002) Long-term impact of preventive proactivity on quality of life of the old-old. Psychosom Med 64:382-394

Kenny DA, Kashy DA, Bolger N (1998) Data analysis in social psychology. In: Gilbert DT, Fiske ST, Lindzey G (eds) The handbook of social psychology, vol I, 4th edn. The McGraw-Hill Companies Inc., Boston, pp 233-265

Kremers IP, Steverink N, Albersnagel FA, Slaets JPJ (2006) Improved self-management ability and well-being in older women after a short group intervention. Aging Ment Health 10:476-484

Lachman ME, Prenda Firth KM (2004) The adaptive value of feeling in control during midlife. In: Brim OG, Ryff CD, Kessler RC (eds) How healthy are we? A national study of well-being at midlife. The University of Chicago Press, Chicago, pp 320-349

Lawton MP (1984) The varieties of well-being. In: Malatesta CZ, Izard CE (eds) Emotion in adult development. Sage, Beverly Hills, pp 67-84

Lerner RM, Busch-Rossnagel A (eds) (1981) Individuals as producers of their development: a life-span perspective. Academic Press, New York

Lucas RE, Diener E, Suh EM (1996) Discriminant validity of subjective well-being measures. J Pers Soc Psychol 71:616-628

Nieboer A, Lindenberg S (2002) Substitution, buffers, and subjective wellbeing: a hierarchical approach. In: Gullone R, Cummins RA (eds) The universality of subjective wellbeing indicators. Kluwer, Dordrecht, pp 175-189 
Picavet HSJ (2001) National health surveys by mail or home interview. Effects on response. J Epidemiol Community Health $55: 408-413$

Rothermund K, Brandtstädter J (2003) Coping with deficits and losses in later life: from compensatory action to accommodation. Psychol Aging 18:896-905

Schuurmans H, Steverink N, Frieswijk N, Buunk BP, Slaets JPJ, Lindenberg S (2005) How to measure self-management abilities in older people by self-report. The development of the SMAS30. Qual Life Res 14:2215-2228

Sobel ME (1982) Asymptotic confidence intervals for indirect effects in structural equations models. In: Leinhardt S (ed) Sociological methodology 1982. Jossey-Bass, San Francisco, pp 290-312

Steverink N, Lindenberg S (2006) Which social needs are important for subjective well-being? What happens to them with aging? Psychol Aging 21:281-290
Steverink N, Lindenberg S, Slaets JPJ (2005) How to understand and improve older people's self-management of wellbeing. Eur J Ageing 2:235-244

Thompson SC (2002) The role of personal control in adaptive functioning. In: Snyder CR, Lopez SJ (eds) Handbook of positive psychology. Oxford University Press, Oxford, pp 202215

Watson D, Clark LA, Tellegen A (1988) Development and validation of brief measures of positive and negative affect: the PANAS scales. J Pers Soc Psychol 54:1063-1070

Wrosch C, Heckhausen J, Lachman ME (2000) Primary and secondary control strategies for managing health and financial stress across adulthood. Psychol Aging 15:387-399 